\title{
El papel de la comunicación en la gestión de las situaciones de crisis desde las administraciones públicas
}

\author{
Ana Alcantud \\ Ecoinstitut Barcelona \\ ana.alcantud@ecoinstitut.es \\ Dafne Mazo \\ Ecoinstitut Barcelona \\ dafne.mazo@ecoinstitut.es
}

\begin{abstract}
Resumen
En un contexto de crisis, las administraciones públicas juegan un papel crucial a la hora de gestionar los recursos. Durante el episodio de sequía vivido en Cataluña entre 2007 y 2008, se aprobaron instrumentos legales que establecían las bases para la promoción de campañas comunicativas orientadas a implicar a los consumidores en un uso racional del agua, con el fin de poder garantizar el suministro doméstico y así poder cubrir sus necesidades vitales. El artículo se centra en el análisis de las actividades comunicativas promovidas por las administraciones autonómicas y locales. Se analiza su funcionamiento, efectos e impacto ambiental tanto en términos de cambio de hábitos de la población como en términos cuantitativos de efectividad en el ahorro doméstico de agua. Asimismo, se quiere determinar cuáles son los elementos clave de la comunicación institucional para promover una gobernanza que incremente la complicidad de la ciudadanía, aumente la efectividad de las actividades promovidas en el ámbito de la sostenibilidad y sirva para legitimar la intervención de las administraciones públicas en ámbitos privados como el del consumo, en pos de la promoción de la sostenibilidad y el uso racional de los recursos naturales, escasos y finitos.
\end{abstract}

Palabras clave Comunicación, consumo sostenible, gestión de la crisis, gobernanza, legitimidad, sequía, transparencia.

\section{The role of communication as a tool for Public Administrations to manage crisis situations}

\begin{abstract}
Within a crisis context public administrations play a key role when it comes to managing resources. During the drought period lived in Catalonia between the years 2007 and 2008, legal instruments where passed setting the grounds for the promotion of communicative campaigns directed toward the implication of consumers in the rational use of water, guaranteeing the domestic supply in order to cover vital needs. The article focuses on the analysis of the communicative activities promoted by local and regional administrations. The functioning, the effects and the resulting environmental impacts are analysed in terms of population's behaviour changes as well as in quantitative terms of domestic water savings. Furthermore, it will be determined which are the key elements for an efficient institutional communication when aiming to promote a governance that encourages complicity with citizens, increases the efficiency of the activities promoted within the sustainability context and is used for legitimating public administrations' intervention regarding private areas such as consumption, in favor of the promotion of sustainability and the rational use of scarce and finite natural resources.
\end{abstract}




\section{INTRODUCCIÓN}

La climatología mediterránea inherente a los países del sur de Europa se caracteriza por la alternancia de periodos de lluvias abundantes con períodos de sequía (MED WS\&D WG, 2007). Por lo tanto, la cíclica escasez hídrica obliga a las administraciones públicas (AAPP) a hacer una gestión sostenible del agua, consistente en conservar los recursos hídricos para poder garantizar el suministro en épocas de sequía. De igual manera, el uso racional del agua es algo familiar para la población catalana y es el vector ambiental hacia el cual la población es más sensible, a diferencia de otros como los residuos o la energía. No necesariamente porque haya una mayor concienciación ambiental vinculada al consumo del agua, sino porque la escasez de agua se puede percibir como una amenaza para la supervivencia.

En este contexto general, en 2005 y 2007 se sucedieron dos importantes periodos de sequía en Cataluña. Por este motivo, en 2005, el Gobierno catalán aprobó el Decreto de Sequía 93/2005, que fue elaborado con el fin de regular el uso del agua y preservar la disponibilidad del suministro de la misma durante el mayor tiempo posible. Las medidas de este decreto se centraron principalmente en la aplicación de progresivas restricciones en los suministros urbanos.

La sequía de 2007 provocó una nueva situación de crisis, cuyo resultado fue el Decreto de Sequía 84/2007, que tenía muchas similitudes con el anterior decreto, pero que implicaba una mejora en cuanto a la previsión de los aspectos comunicativos y requería otras medidas adicionales para preservar el suministro a los sectores doméstico y productivo el máximo tiempo posible.

Así, durante el período de sequía de 2007-2008 la administración pública catalana (tanto autonómica como local) realizó un gran esfuerzo de gestión conjunta de la crisis que se concentró en dos tipos de actividades diferenciadas:

Acelerar todas aquellas obras de infraestructura que garantizasen un mejor uso del agua potable. Es decir, se hizo un esfuerzo importante por mejorar la red de suministro de agua potable y por establecer las infraestructuras necesarias para utilizar aguas freáticas para fines ajenos al consumo humano (limpieza de calles, fuentes ornamentales, riego de jardines y parques públicos, etc.), reservando el agua potable para el consumo humano.

Comunicar la necesidad de hacer un uso racional y ahorrador del agua en los sectores doméstico y productivo.

El presente artículo se centra en este segundo grupo de actividades, más concretamente en el análisis de las actividades comunicativas promovidas por las administraciones autonómicas y locales. Se analiza su funcionamiento, efectos e impacto ambiental tanto en términos de cambio de hábitos de la población como en términos

176 cuantitativos de efectividad en el ahorro doméstico de agua. 
Las actividades comunicativas se consideran elementos fundamentales para la gobernanza. La gobernanza es la noción de que, en las condiciones de la sociedad contemporánea (nacional e internacional), el gobierno es un agente de dirección necesario pero insuficiente aun si dispusiera de todas las capacidades requeridas. Las definiciones del concepto destacan además las formas de diálogo y negociación que practican los actores públicos y privados para ajustar sus intereses, conciliar sus diferencias y llegar a los acuerdos básicos que serán el marco de referencia para elaborar las políticas y prestar los servicios públicos de beneficio social o para decidir políticas estratégicas y proyectos de inversión cruciales para la viabilidad y prosperidad de la sociedad (Aguilar y Bustelo, 2010).

La desterritorialización de la economía, la complejidad de la sociedad y la fragmentación del conocimiento hacen del gobierno uno de los agentes requeridos para la dirección de la sociedad. Esto conlleva una nueva relación entre gobierno y sociedad, que difícilmente puede ser ya en modo de mando y control. En este sentido, gobernanza significa el cambio de proceso/modo/patrón de gobierno: el paso de un centro a un sistema de gobierno, en el que se requieren y activan los recursos del poder público, de los mercados y de las redes sociales. En conexión, es el paso de un estilo jerárquico a un estilo de gobernar asociado e independiente entre organismos gubernamentales, organizaciones privadas y sociales (Aguilar, 2007).

La gobernanza es un elemento clave de la sostenibilidad. El desarrollo sostenible sólo es viable desde la perspectiva relacional en tanto que los impactos de las políticas públicas no dependen única y exclusivamente de la acción gubernamental sino que también de la adquisición de hábitos y comportamientos sostenibles por parte de ciudadanía y sectores productivos que sólo pueden ser voluntariamente asumidos. Esto es especialmente importante en el ámbito del consumo como por ejemplo en el caso del consumo doméstico de agua (Aguilar y Bustelo, 2010).

Así, desde esta perspectiva relacional, es necesario que la ciudadanía sea partícipe de las iniciativas públicas y conozca los efectos ambientales de sus propias acciones. De este modo las políticas que promueven el consumo sostenible no se percibirán como una intromisión en la vida privada.

En el caso del consumo doméstico de agua, el uso de instrumentos coercitivos sólo se justifica por la situación de crisis creada por la sequía. En cualquier otro contexto, la administración sólo puede utilizar instrumentos comunicativos que convenzan de la necesidad de hacer un consumo responsable de agua en el hogar. En este sentido, la comunicación de los resultados ambientales de la acción pública en términos de ahorro de agua es básica para legitimar la intervención pública en el ámbito privado.

Es además sumamente importante la transparencia. Es necesario que los ciudadanos se sientan constantemente informados de cuáles son las medidas que se toman, con qué recursos cuentan las AAPP, cuáles son los motivos que les mueven a 
actuar de una manera y no de otra, qué beneficios se obtienen tras implantar y llevar a cabo dichas medidas, etc. Y como último requisito, los resultados obtenidos deben ser comunicados y estar accesibles para los ciudadanos. La rendición de cuentas dota de sentido a las decisiones tomadas y los esfuerzos exigidos a la ciudadanía.

A lo largo del artículo se analizan las actividades comunicativas de las AAPP en el contexto de la sequía y sus efectos en el ámbito del consumo sostenible y cambio de hábitos de la ciudadanía. Pero también se estiman los impactos ambientales de la campaña “iInstálame!: cada gota cuenta”. El objetivo es poder determinar cuáles son los elementos clave de la comunicación para promover una gobernanza que incremente la complicidad de la ciudadanía, aumente la efectividad de las actividades promovidas en el ámbito de la sostenibilidad y se reconozca la legitimidad de las AAPP para intervenir en ámbitos privados como el del consumo, en pos de la promoción de las sostenibilidad y el uso racional de los recursos naturales, escasos y finitos.

\section{LAS ACTIVIDADES COMUNICATIVAS PÚBLICAS DURANTE EL PERIODO DE SEQUÍA}

El Decreto de Sequía 84/2007 y la Moción Parlamentaria 21/VIII fueron, durante el episodio de sequía de 2007-2008, los dos principales mecanismos de gobernanza y establecían las bases de las medidas necesarias para gestionar los escasos recursos hídricos en este tipo de situaciones. Entre las diferentes medidas propuestas estaban las actividades comunicativas orientadas a implicar a los consumidores particulares, siendo por si mismos parte del problema pero también de su solución.

La reducción efectiva del consumo de agua se obtiene mediante el aumento de la concienciación ciudadana sobre el valor que tiene el agua como recurso escaso que debe ser preservado y empleado de forma racional. Una de las campañas previstas por la Moción Parlamentaria 21/VIII fue la Campaña “iInstálame!: cada gota cuenta” cuyo principal objetivo era promover la concienciación ciudadana.

La campaña "¡Instálame!: cada gota cuenta” fue uno de los instrumentos comunicativos institucionales diseñados por el Gobierno catalán (entre el Departamento de Presidencia, el Departamento de Medio Ambiente y la Agencia Catalana de Agua$\mathrm{ACA}$ ), en colaboración con la asociación medioambiental "Ecologistes en Acció" y varias asociaciones de ferreteros y cadenas de distribución.

La campaña consistió en la distribución gratuita de un "kit" de ahorro de agua para grifos que constaba de dos dispositivos reductores de caudal, denominados "aireadores", y un tríptico divulgativo presentando la situación de emergencia, dando instrucciones de cómo instalar los mismos y explicando cómo identificar (mediante logotipo) a las ferreterías donde se podían comprar los "aireadores". En total se distribuyeron 1.300.000 dispositivos junto con la prensa escrita vendida durante los días

17819 y 20 de enero de 2008. 


\subsection{Estructura de las actividades comunicativas públicas durante la sequía 2007-2008}

Los impactos de las estrategias y políticas de consumo sostenible dependen de una serie de pasos. En primer lugar, las estrategias y políticas se desarrollan en forma de "outputs", como leyes, normas, programas, planes e implementación de medidas. En segundo lugar, estos "outputs" pueden provocar una serie de "outcomes" o efectos en términos de cambios de comportamiento de los ciudadanos (por ejemplo, en la demanda, el uso o deshecho de los productos o servicios). Y por último, los cambios de comportamiento pueden provocar diversos impactos económicos, ambientales o sociales, que pueden tener lugar por medio de cambios en las pautas de producción, en otras pautas de consumo o cambios en las cadenas de producto completas.

\section{GRÁFICO 1}

\section{Estructura de las actividades comunicativas públicas durante el periodo de sequía}

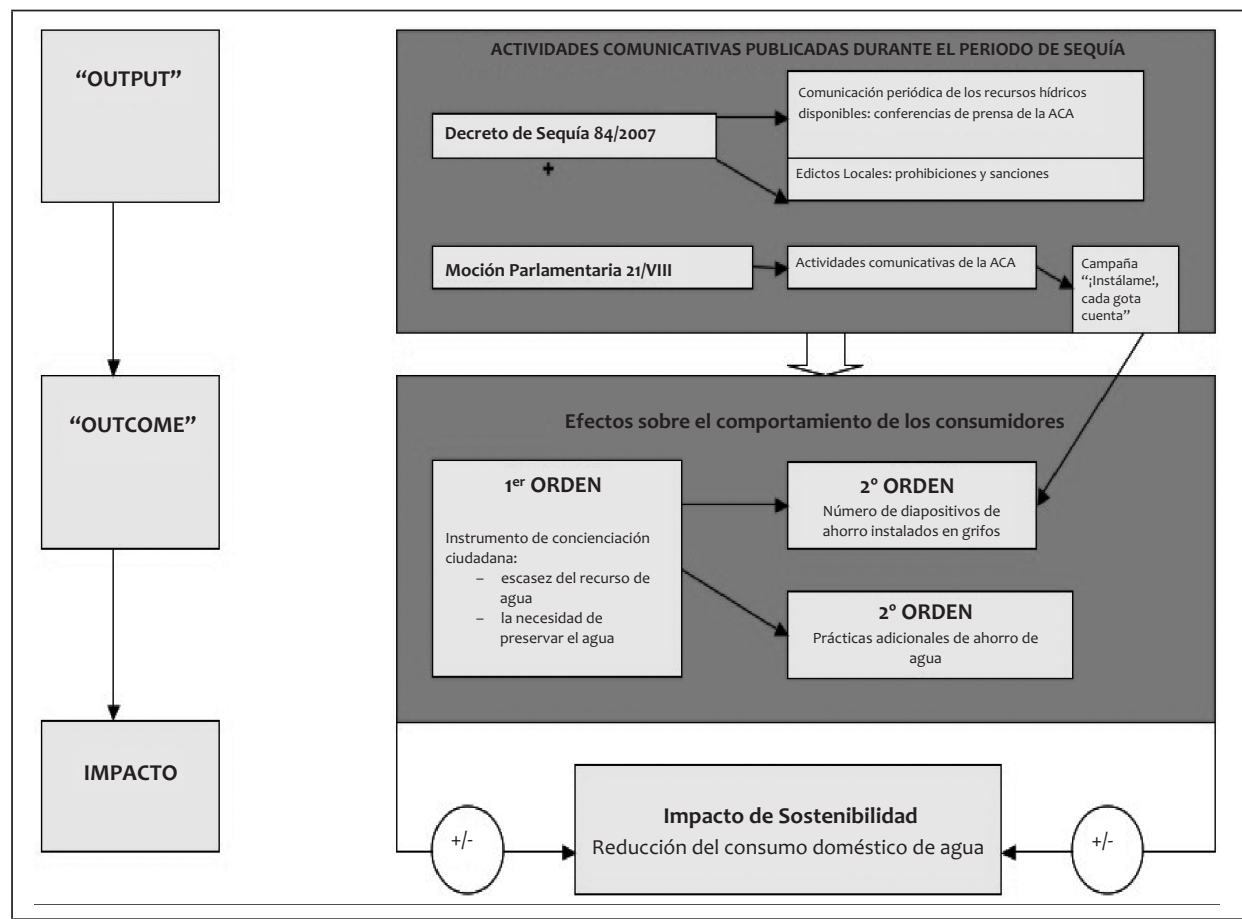

Fuente: Elaboración propia.

\subsubsection{Output}

El output del instrumento se basa principalmente en los dos instrumentos regulativos mencionados con anterioridad: el Decreto de Sequía 84/2007 y la Moción Parlamentaria 21/VIII, que establecen las bases para la elaboración de las medidas adecuadas para gestionar los escasos recursos hídricos en periodos críticos de sequía. 
El Decreto de Sequía preveía la creación de una "Comisión Inter-institucional” que consistía en 6 Departamentos de la Generalitat de Cataluña, la Agencia Catalana de Agua (ACA), la Asociación Catalana de Comunidades y Municipios, la Federación Catalana de Comunidades y la Entidad Metropolitana de Servicios Hidráulicos y Tratamiento de Residuos (EMSHTR)․ La coordinación de la Comisión recaía en la ACA, que establecía las medidas restrictivas.

La ACA y la Comisión Inter-institucional eran las entidades gestoras principales pero la participación de otras AAPP y sus responsabilidades variaban en función de la gravedad de la situación.

Una parte importante del Decreto de Sequía consistía en la definición de escenarios de excepcionalidad: Pre-Alerta, Estado de Excepcionalidad 1, Estado de Excepcionalidad 2 y Estado de Emergencia. Cada uno de estos escenarios, en los que se definían las medidas de gestión aplicables y la administración pertinente para ello, se activaba en función del caudal de las cuencas fluviales y la existencia de recursos hídricos en los embalses.

\section{CUADRO 1}

Principales actuaciones en el marco de los escenarios del Decreto de Sequía

\begin{tabular}{|c|c|}
\hline \multirow[t]{2}{*}{ Pre- Alerta } & - Se intensifica la monitorización de la evolución de los embalses. \\
\hline & - Medidas de información y concienciación. \\
\hline \multirow[t]{4}{*}{$\begin{array}{l}\text { Estado de } \\
\text { Excepcionalidad } 1\end{array}$} & $\begin{array}{l}\text { - Reducción del } 15 \% \text { en el suministro para irrigación en los sistemas } \\
\text { regulados. }\end{array}$ \\
\hline & - Controles intensificados a los usuarios. \\
\hline & $\begin{array}{l}\text { - Intensificación de las medidas de ahorro de agua en las redes de } \\
\text { suministro. }\end{array}$ \\
\hline & $\begin{array}{l}\text { - Limitación del suministro a los municipios (270 litros por habitante } \\
\text { y día). }\end{array}$ \\
\hline \multirow[t]{4}{*}{$\begin{array}{l}\text { Estado de } \\
\text { Excepcionalidad } 2\end{array}$} & $\begin{array}{l}\text { - Reducción del } 45 \% \text { en el suministro para irrigación en los sistemas } \\
\text { regulados. }\end{array}$ \\
\hline & $\begin{array}{l}\text { - Mayor intensificación de las medidas de ahorro de agua en las } \\
\text { redes de suministro. }\end{array}$ \\
\hline & - Restricciones para los usos no básicos. \\
\hline & $\begin{array}{l}\text { - Limitación del suministro a los municipios (230 litros por habitante } \\
\text { y día). }\end{array}$ \\
\hline Emergencia & - Racionamiento del suministro. \\
\hline
\end{tabular}

Fuente: Decreto de Sequía 84/2007

1 EMSHTR es la entidad pública que agrupa a todos los municipios del Área Metropolitana de Barcelona (AMB), es la entidad pública responsable del suministro de agua hasta los depósitos de cabecera municipales. 
Durante el estado de Excepcionalidad 2 quedaba prohibido emplear el agua con fines suntuarios tanto por las autoridades locales como por los usuarios finales. Durante este periodo, la EMSHTR establecía el sistema de prohibiciones y sanciones que eran comunicadas por los municipios a los usuarios finales por medio de edictos o bandos locales. Básicamente se prohibían las siguientes actividades:

- El riego de jardines privados, jardines públicos e instalaciones deportivas (tanto privadas como públicas).

— La limpieza de calles, carreteras y aceras (tanto públicas como privadas).

— El llenado de piscinas, estanques y fuentes (tanto públicas como privadas).

- El lavado de vehículos con mangueras, excepto en el caso de las compañías especializadas en el lavado de coches.

Estas prohibiciones sólo se trasladaron en un par de sanciones y no implicaron grandes sumas de dinero, pero fueron ejemplarizantes porque tuvieron un gran impacto gracias su publicación en prensa.

En cuanto a las actividades comunicativas asociadas a los escenarios de excepcionalidad:

- Durante el Estado de Excepcionalidad 1: se preveía que cada 15 días debía comunicarse la situación de los embalses y los niveles pluviométricos. Así, cada 15 días se realizaba una rueda de prensa y se actualizaba toda la información en la página web de la ACA.

- Durante el Estado de Excepcionalidad 2: cuando las reservas de agua estaban al 22\% de su capacidad, se intensificaron los esfuerzos de comunicación de las autoridades públicas. La ACA emitía una rueda de prensa cada semana y se Ilevaba a cabo por el Consejero de Medio Ambiente de la Generalitat de Cataluña.

Paralelamente a las actividades comunicativas vinculadas al Decreto 84/2007, la ACA promovió 9 actividades comunicativas de acuerdo con el despliegue de la Moción Parlamentaria 21/VIII. Los principales canales de comunicación fueron los anuncios en TV/radio, prensa, transportes públicos, además de panfletos y pósteres. Una de las actividades programadas, tal y como se ha mencionado anteriormente, fue la Campaña “iInstálame!: cada gota cuenta”, que tuvo lugar en un contexto de numerosas actividades comunicativas destinadas a llegar a los ciudadanos a través de las autoridades públicas. 
CUADRO 2

Actividades comunicativas (Moción Parlamentaria 21/VIII)

\begin{tabular}{|c|c|c|}
\hline Año & Actividad comunicativa & $\begin{array}{l}\text { Diseminación en los } \\
\text { medios }\end{array}$ \\
\hline Febrero 2007 & $\begin{array}{l}\text { Campaña de sensibilización ciudadana sobre la } \\
\text { sequía y el ahorro de agua: "Si quieres agua, } \\
\text { cierra el grifo" }\end{array}$ & $\begin{array}{l}\text { - Anuncios en radio y TV } \\
\text { - Prensa }\end{array}$ \\
\hline Julio 2007 & $\begin{array}{l}\text { Refuerzo de la campaña de sensibilización: "Si } \\
\text { quieres agua, cierra el grifo" }\end{array}$ & $\begin{array}{l}\text { - Anuncios en radio } \\
\text { - Anuncios en transpor- } \\
\text { tes públicos (metro, au- } \\
\text { tobús, etc.) }\end{array}$ \\
\hline Diciembre 2007 & $\begin{array}{l}\text { Anuncio “Queda poca”: sobre las actuaciones } \\
\text { de gestión de la sequía en funcionamiento por } \\
\text { esas fechas }\end{array}$ & - Prensa \\
\hline Enero 2008 & Campaña “¡Instálame!:cada gota cuenta” & - Prensa \\
\hline Marzo 2008 & $\begin{array}{l}\text { X Fiesta del Agua: Todas las actividades fueron } \\
\text { conceptualizadas por la ACA y orientadas a la } \\
\text { concienciación sobre la escasez de agua y la ne- } \\
\text { cesidad de ahorro: } \\
\text { Manifiesto conjunto de todas las instituciones, } \\
\text { entidades y empresas participantes } \\
\text { Talleres pedagógicos sobre agua } \\
\text { Juegos infantiles con la temática de ahorro de } \\
\text { agua } \\
\text {... }\end{array}$ & $\begin{array}{l}\text { - } 25.000 \text { panfletos distri- } \\
\text { buidos en escuelas } \\
\text { - A6.000 pósteres en } \\
\text { tiendas } \\
\text { - Anuncios en transpor- } \\
\text { tes públicos } \\
\text { - Spots en TV y radio } \\
\text { - Prensa }\end{array}$ \\
\hline $\begin{array}{l}\text { Marzo-abril } \\
2008\end{array}$ & $\begin{array}{l}\text { Campaña "Juntos podemos hacer frente a la } \\
\text { sequía" }\end{array}$ & $\begin{array}{l}\text { - Spots en TV y radio } \\
\text { - Prensa }\end{array}$ \\
\hline $\begin{array}{l}\text { Abril-mayo } \\
2008\end{array}$ & $\begin{array}{l}\text { Anuncio "Estamos trabajando para garantizar } \\
\text { el agua para todos" }\end{array}$ & - Prensa \\
\hline Junio 2008 & $\begin{array}{l}\text { Exposición: “ Damos soluciones para garanti- } \\
\text { zar el agua del futuro” }\end{array}$ & $\begin{array}{l}\text { - Centro de Exposiciones } \\
\text { de la Generalitat Palau } \\
\text { Robert }\end{array}$ \\
\hline Julio 2008 & $\begin{array}{l}\text { Reedición de la Campaña “Juntos podemos ha- } \\
\text { cer frente a la sequía” }\end{array}$ & $\begin{array}{l}\text { - Spot de TV y radio } \\
\text { - Prensa }\end{array}$ \\
\hline
\end{tabular}

Fuente: Sesión de control del Parlamento de Cataluña. Moción Número 390-00021/08 (BOPC número 278; Lunes, 9 de junio de 2008).

Adicionalmente a las actividades comunicativas institucionales, durante la se182 de la situación: 


\section{1) La influencia de los medios de comunicación}

Los medios de comunicación cubrían la situación de crisis como una de las principales noticias durante el periodo de sequía. Había momentos en los que todos los días, las noticias regionales tenían como principal titular el nivel de reserva de agua en los embalses. Además, los periódicos también dieron mucha importancia a la situación de sequía.

\section{2) El cierre de fuentes públicas ornamentales}

Una de las acciones más visibles que provocó impacto sobre la población fue el cierre de fuentes ornamentales públicas. Tuvo lugar durante el Estado de Excepcionalidad 2. Se colocaron carteles informando de por qué se clausuraban las fuentes. Fue una acción muy visible, independientemente de que no resultase relevante en cuanto a ahorro de agua.

\section{3) La llegada de buques con agua desde Marsella}

Con el fin de garantizar el suministro de agua en Barcelona y el Área Metropolitana, el Gobierno catalán compró agua a Francia. Los costes de la misma fueron extremadamente elevados (5-6€/m3 de agua). La capacidad de cada uno de los buques era extremadamente limitada ya que cada uno de ellos sólo podía transportar agua para medio día en la ciudad de Barcelona.

El hecho de que se tuviese que comprar agua y transportarla hasta Barcelona reforzó la percepción de que "no había agua” y creó un sentimiento de preocupación y ansiedad entre los ciudadanos.

\subsubsection{Efectos (Outcomes)}

En cuanto a los efectos o outcomes, el principal efecto perseguido era la promoción de la concienciación de los ciudadanos acerca de la escasez del recurso hídrico y la necesidad de preservarlo. En este sentido la campaña “instálame!: cada gota cuenta", junto con el resto de las campañas y actividades institucionales promovidas por los entes públicos, contribuyó a incrementar esta concienciación. De acuerdo con un estudio realizado por la ACA para evaluar la efectividad de la campaña, el 91\% de los entrevistados consideraron que la distribución gratuita de dispositivos de ahorro de agua contribuyó al aumento de la concienciación hacia un mayor ahorro del consumo de agua (ACA, 2008).

Un segundo efecto que puede prácticamente atribuirse directa e íntegramente a la referida campaña es la instalación real de los "aireadores" con reductor de caudal 
en los grifos domésticos. En este sentido, la campaña ofreció una solución real para hacer frente a la preocupación sobre la escasez de agua de los ciudadanos. El $61 \%$ de los entrevistados instaló al menos un dispositivo de ahorro en uno de los grifos de su hogar, el $47 \%$ instaló dos mecanismos y el $7 \%$ restante lo intentó pero no funcionó (ACA, 2008). Estos dispositivos fueron percibidos como mecanismos eficientes para ahorrar agua y al mismo tiempo contribuyeron a reducir en los hogares los gastos relativos al agua.

El tercer efecto que se puede vincular a la campaña "¡Instálame!: cada gota cuenta" son las prácticas de ahorro doméstico de agua que se pusieron en práctica de forma adicional a la instalación de los dispositivos. De acuerdo con el lema de la campaña fueron muchos los ciudadanos que literalmente lo pusieron en práctica y extremaron las medidas de ahorro.

De acuerdo con la encuesta "Hogares y Medio Ambiente. 2008" (INE, 2008), el 94,1\% de los hogares catalanes pusieron en práctica comportamientos de ahorro de agua como: reutilizar el agua, utilizar el lavavajillas empleando la capacidad al máximo, cerrar el grifo para lavarse los dientes o ducharse en lugar de bañarse.

Sin embargo, a pesar de los resultados positivos promovidos por la campaña “innstálame!: cada gota cuenta”, durante el periodo de crisis originado por la sequía hubo cierta controversia entre el gobierno y la oposición acerca de las medidas tomadas para gestionar la crisis. En algunos casos, los ciudadanos entendieron las numerosas campañas y acciones como publicidad institucional, con lo que asumieron que la responsabilidad de preservar el recurso durante el mayor tiempo posible recaía en ellos mismos.

\subsubsection{Impacto ambiental estimado de la campaña "instálame!: cada gota cuenta"}

El principal impacto ambiental que quería lograrse con la distribución gratuita de mecanismos de ahorro para los grifos de la campaña era la reducción del consumo doméstico de agua. Durante el peor periodo de sequía (con Estado de Excepcionalidad 2) se alcanzaron ahorros de alrededor del 20\%. Sin embargo, no pueden atribuirse estos ahorros exclusivamente a la campaña.

Es necesario analizar los cambios en el consumo doméstico a largo plazo debido a los siguientes motivos:

- el hecho de que se distribuyesen "aireadores" para los grifos no significa que todos los dispositivos fuesen instalados;

- incluso aunque los dispositivos fueran instalados no significa que se hiciese 184 de forma inmediata; 
- los valores pueden fluctuar debido a diferentes factores (el número de personas en el hogar a causa de las vacaciones y otros motivos), con lo cual los valores obtenidos podrían ser inferiores a los reales.

También hay que tener en cuenta que los efectos del ahorro de agua son más duraderos que la propia campaña o el periodo de sequía, ya que una vez superado el estado de emergencia no se desinstalan los "aireadores" reductores de caudal de los grifos.

A pesar de estas afirmaciones se puede hacer una estimación del impacto ambiental en términos de ahorro de agua tomando como base el estudio de la ACA (2008). Dicho estudio es una encuesta estadísticamente representativa finalizada en marzo de 2008, entre cuyos principales resultados destaca que el $47 \%$ de los encuestados había instalado los dos "aireadores" reductores de caudal distribuidos en la campaña y un 14\% había instalado tan sólo uno. Teniendo en cuenta que estos son los resultados del estudio un mes después de finalizar la campaña, y que desconocemos si hay más ciudadanos que instalaron los "aireadores" después, sólo podemos basarnos en las siguientes asunciones para estimar el impacto ambiental en términos de ahorro de agua:

1 Se consideran válidos los resultados del estudio: asumiendo que el estudio es fiable y que los encuestados no mintieron.

2. Considerando que al menos uno de los dispositivos fue instalado en el $61 \%$ de los 650.000 hogares y asumiendo que por hogar sólo se compró un periódico, 403.000 hogares instalaron al menos un dispositivo de reducción de caudal.

3. Los ahorros potenciales de un "kit" (con dos mecanismos de ahorro) están entre $12-15 \%$ del consumo de un hogar. Por lo tanto, se considera que la instalación de un solo aireador con reductor de caudal en un grifo del baño o de la cocina genera un ahorro entre un 6 y un 7,5\% (Alcantud y Mazo, 2010) ${ }^{2}$.

La información sobre el consumo de agua en los hogares proporcionada por la ACA está basada en las facturas de consumo agua de las empresas suministradoras.

Tomando como base la información proporcionada por la ACA sobre el consumo de agua en los hogares en 2007 y aplicando el intervalo de reducción mencionado del 6-7,5\%, el impacto podría ser el siguiente:

2 Datos obtenidos por medio de entrevistas realizadas a expertos para la elaboración del documento Impact Assessment Paper: The "Install me!'" Campaign. Communicative campaign within the Drought Context in Catalonia, en el marco del proyecto del $7{ }^{\circ}$ Programa Marco de la UE EUPOPP Policies to promote sustainable consumption patterns. 
TABLA 1

Estimación de impacto (1) de la Campaña "ilnstálame!: cada gota cuenta”

\begin{tabular}{|l|c|c|c|}
\hline \multicolumn{1}{|c|}{ Estimación de impacto } & $\begin{array}{c}\text { Número } \\
\text { de } \\
\text { hogares }\end{array}$ & $\begin{array}{c}\text { Ahorro de agua en los } \\
\text { hogares en } \mathrm{m}^{3} / \text { año } \\
\text { (Asunción del 6\%) }\end{array}$ & $\begin{array}{c}\text { Ahorro de agua en los } \\
\text { hogares en } \mathrm{m}^{3} / \text { año } \\
\text { (Asunción del 7’5\%) }\end{array}$ \\
\hline $\begin{array}{l}\text { Instalación de un dispositivo } \\
\text { de reducción de caudal (14\% } \\
\text { de los hogares) }\end{array}$ & 91.000 & 821.703 & 1.027 .129 \\
\hline $\begin{array}{l}\text { Instalación de dos dispositi- } \\
\text { vos de reducción de caudal } \\
\text { (47\% de los hogares) }\end{array}$ & 312.000 & 5.634 .534 & 7.043 .167 \\
\hline Total & 403.000 & 6.456 .236 & 8.070 .296 \\
\hline
\end{tabular}

Fuente: Elaboración propia a partir de datos facilitados por la ACA.

Se estima que entre 2007 y 2008 se obtuvo una reducción del consumo de agua en los hogares de 10.116.904 m3, es por tanto posible atribuir un 65-80\% de esta reducción a la campaña “iInstálame!: cada gota cuenta”.

TABLA 2

Estimación de impacto (2) de la Campaña "instálame!: cada gota cuenta”

\begin{tabular}{|l|c|c|}
\hline $\begin{array}{c}\text { Reducción del consumo de agua en los hogares debido } \\
\text { a la campaña “ilnstálame!: cada gota cuenta” }\end{array}$ & $\mathbf{m}^{3}$ & $\%$ \\
\hline Ahorro de agua en los hogares (Asunción 12\%) & 6.456 .236 & $65 \%$ \\
\hline Ahorro de agua en los hogares (Asunción 15\%) & 8.070 .296 & $80 \%$ \\
\hline Ahorro total de agua en los hogares (2008) & $\mathbf{1 0 . 1 1 6 . 9 0 4}$ & $\mathbf{1 0 0 \%}$ \\
\hline
\end{tabular}

Fuente: Elaboración propia a partir de datos facilitados por la ACA.

El impacto ambiental de la instalación de los mecanismos de ahorro de agua en los grifos perdura más allá del contexto de sequía porque una vez instalados no se desinstalan cuando se supera el estado de crisis. Algunos expertos afirman que los dos últimos episodios de sequía (2005 y 2007-2008) hicieron cambiar la pauta del consumo doméstico de agua (Alcantud y Mazo, 2010). La tendencia actual consiste en que se reduce el consumo doméstico de agua durante los periodos de sequía y en períodos de no escasez se mantiene el mismo consumo, no se vuelven a recuperan los niveles de consumo anteriores a la crisis. De manera que, por ejemplo, en el Área Metropolitana de Barcelona ya hay municipios que consumen menos de 100 litros de agua/habitante/día y los expertos afirman que se desconoce hasta qué punto puede reducirse el consumo doméstico de agua.

Según declaraciones en prensa de Francesc Narváez, presidente de la Entidad de Medioambiente del Área Metropolitana (l'Entitat del Medi Ambient, EMA), la reducción progresiva del consumo doméstico de agua muestra el conocimiento de los ciu- 
dadanos, así como su implicación. Aunque durante los años 2009 y 2010 las lluvias hayan sido abundantes el nivel de consumo de agua ha continuado bajando.

El Área Metropolitana de Barcelona $(A M B)$ es una de las áreas de Europa con menor nivel de consumo de agua (ACA, 2008). De acuerdo con la EMA, en el año 2000 el nivel de consumo fue de 132,8 litros por habitante y día, lo que implica que en los 10 años transcurridos hasta el 2010 el consumo se redujo en un 19\% (AMB, 2011). Los mayores porcentajes de reducción se alcanzaron durante los dos últimos periodos de sequía (2005 y 2007-2008).

TABLA 3

Reducción del consumo doméstico en el periodo 2005-2010

\begin{tabular}{|c|c|c|}
\hline Año & litro/habitante/día & Decrecimiento Anual \\
\hline $\mathbf{2 0 0 6}$ & 116,6 & - \\
\hline $\mathbf{2 0 0 7}$ & 113,8 & $-2,4 \%$ \\
\hline $\mathbf{2 0 0 8}$ & 109,9 & $-3,4 \%$ \\
\hline $\mathbf{2 0 0 9}$ & 108,4 & $-1,4 \%$ \\
\hline $\mathbf{2 0 1 0}$ & 107,4 & $-0,9 \%$ \\
\hline
\end{tabular}

Fuente: AMB, 2010.

Según los datos de la EMA, el nivel de consumo de 2010 en el AMB (107,4 litros por habitante y día) fue muy bajo comparado con los 273 litros por habitante y día de Estocolmo, los 289 litros por habitante y día de Génova, los 158 litros por habitante y día de Londres o los 131 litros por habitante y día de Madrid.

\section{CONCLUSIONES}

El Decreto de Sequía 84/2007 fue derogado por el Decreto 5/2009 del 13 de enero de 2009 , debido al incremento de las reservas de agua provocado por las lluvias. Sin embargo, el consumo doméstico de agua se mantuvo a niveles bajos. Algunos municipios del AMB (Badia del Vallès, L'Hospitalet de Llobregat y Cornellà del Llobregat) alcanzaron en 2008 niveles de consumo de agua inferiores a 100 litros por habitante y día (AMB, 2008), que son los niveles establecidos por la Organización Mundial de la Salud para cubrir las necesidades básicas.

Entre otras cosas, estos buenos resultados, que reflejan el éxito de las acciones comunicativas promovidas por las AAPP, son debidos al acertado diseño de estas acciones. El mensaje principal que se quiso transmitir fue “¿Cierra el grifo!”, y este mensaje perdura en el tiempo debido a los periódicos episodios de sequía que se suelen suceder en algunas regiones. Además, para alcanzar una elevada repercusión se distribuyeron los "kits de ahorro de agua" con los principales diarios. Un elemento clave de estos "kits" era que los dispositivos de disminución de caudal se distribuían 
de manera gratuita, eran fáciles de usar y la colocación de los mismos no requería ningún sacrificio ni implicaba modificaciones significativas en los hábitos diarios de los ciudadanos.

Otra clave del éxito de las acciones promovidas por las AAPP consistió en que éstas estaban coordinadas con el fin de evitar el solapamiento de campañas. La interacción con el gremio de los ferreteros también resultó de suma importancia. Y a todo ello hay que añadir que, debido a la situación de crisis provocada por la escasez del recurso hídrico, todo tuvo lugar "en el lugar adecuado y el momento adecuado". Los efectos positivos puestos de manifiesto por medio de cambios en los hábitos de consumo, uso racional del agua y reducción del consumo doméstico, fueron resultado de la "afinidad electiva"3. El contexto de sequía y los instrumentos públicos interactuaron reforzándose mutuamente y provocando efectos duraderos que se mantienen una vez la situación de crisis finaliza. Es por ello que los efectos no pueden atribuirse a una sola campaña, sino que hay que tener en cuenta todas las acciones y medidas promovidas por las AAPP dentro del contexto de sequía como promotoras de un consumo sostenible del agua.

La coordinación entre actores públicos y privados en la gestión de la comunicación es un ejemplo clave de cómo desde la gobernanza se obtienen mejores resultados que actuando independientemente.

De forma adicional a los factores que influyeron en el éxito de la gestión de la situación de crisis, un análisis de las acciones públicas llevadas a cabo durante el periodo 2007-2008 pone de manifiesto que durante todo el proceso hubo un esfuerzo por la transparencia. Las AAPP implicadas en la gestión de la crisis (la ACA, la EMSHTR y los entes locales) hicieron un gran esfuerzo de comunicación continua tanto del estado de las existencias de recursos hídricos y las obras para mejorar el suministro como de la necesidad de ahorrar agua.

Sin embargo, no hubo suficiente comunicación en cuanto a los resultados obtenidos tras las numerosas acciones y campañas llevadas a cabo. No se le dio la importancia necesaria a la comunicación de los resultados y al impacto ambiental asociado, para lo cual hubiese hecho falta generar indicadores que permitiesen la cuantificación del impacto en términos de ahorro o beneficios ambientales. En el caso que nos ocupa, se trataría de evaluar en qué medida las campañas de comunicación sobre ahorro de agua infieren al cambio de hábitos y al ahorro efectivo de agua en términos cuantitativos (litros/persona/día). La generación de indicadores de impacto ambiental de políticas públicas puede suponer dos retos:

1. Vincular la acción política al impacto ambiental de una manera pertinente. En el caso de las políticas de agua es evidente que el principal impacto es el

3 Empleamos la expresión de Max Weber: Wahlverwandtschaft. 
ahorro del agua. Sin embargo, frecuentemente se intenta hacer el esfuerzo de traducir el impacto de determinadas políticas ambientales (como la compra pública verde) en un único indicador ( $\left.\mathrm{CO}_{2}\right)$, cuando hay otros más vinculados a la acción política concreta y son más comprensivos y cercanos, lo cual facilita su comunicabilidad.

2. Definir un indicador que sea operativo y del cual se puedan obtener datos de manera continuada y con relativa facilidad. Las políticas públicas en el ámbito de la sostenibilidad frecuentemente buscan reducir el impacto ambiental de la actividad humana; por lo tanto, es necesario definir indicadores de impacto medibles a lo largo del tiempo, y deben ser relativamente sencillos para permitir que los responsables de la política en cuestión puedan calcular y transmitir los resultados.

La comunicación de estos resultados sería fundamental para la legitimación de las acciones emprendidas por las AAPP y acabaría con el sentimiento que tienen algunos ciudadanos de que las campañas no fueron más que "propaganda institucional". Parte de la ciudadanía, principalmente debido a la importante controversia política que se generó entre el Gobierno de la Generalitat y la oposición en cuanto a la gestión de la sequía llevada a cabo por las AAPP, interpretó las campañas informativas como "publicidad institucional" y asumió que era cada uno como ciudadano quien debía tomar las medidas adecuadas para garantizar el ahorro de agua. Esto se puede interpretar como un proceso de empoderamiento de la población como reacción a lo que interpretaban como una mala gestión y "publicidad institucional”.

La evaluación justificaría la intromisión en la vida privada de los ciudadanos así como el uso de instrumentos coercitivos (prohibiciones y multas) en el contexto de crisis. El problema de legitimación va normalmente asociado a cuestiones relativas al consumo sostenible. En esta área, las AAPP sólo pueden sugerir, informar, estimular, etc. Utilizar instrumentos coercitivos en el ámbito privado doméstico no se considera legítimo. Sólo es tolerable en casos de crisis.

La evaluación es además un elemento clave del sistema actual de gobernanza, a través de la cual el gobierno no es el único agente de decisión sino que adquiere un carácter más participativo, implicando a actores públicos y privados para ajustar sus intereses. A la evaluación se le reconocen 3 funciones claras (Aguilar y Bustelo, 2010):

- Aprendizaje o mejora: el papel es informar sobre las potencialidades y dificultades de una determinada acción, con el fin de utilizar dicha información para introducir cambios y mejoras en dicha acción.

- Rendición de cuentas: rendir cuentas a la ciudadanía y a los actores involucrados sobre cómo se han empleado los fondos públicos, cómo se ha interpretado el interés general y cómo se ha dado (o intentado dar) solución a las diversas demandas de la ciudadanía. 
- Iluminar acciones futuras: la evaluación contribuye a un aprendizaje a más largo plazo y que trasciende a las enseñanzas sobre una acción concreta "iluminando" así la acción pública general, el mayor conocimiento y comprensión de la realidad y las mejores maneras y estrategias generales para actuar sobre la misma.

Estas tres funciones, claves para el éxito del sistema de gobernanza, es importante tenerlas en cuenta a la hora de elaborar un sistema de gestión para hacer frente a cualquier tipo de situación, incluida una situación de crisis en relación a recursos naturales básicos. En el caso de la gestión de la situación de sequía, hubiese sido interesante hacer hincapié en la rendición de cuentas en términos de disminución del consumo doméstico y público de agua. Hubiese sido el último elemento para completar el rompecabezas y asegurar así el reconocimiento de la legitimidad de las AAPP en las acciones promovidas en la crisis, ya que gracias a la acción y el esfuerzo de ciudadanía y AAPP el consumo doméstico medio alcanzado en el Área Metropolitana de Barcelona en el 2010 fue de 107,4 litros por habitante y día (AMB, 2011), lo que sitúa a Barcelona y su Área Metropolitana en el área urbanizada europea con el menor consumo de agua por habitante y día.

\section{REFERENCIAS BIBLIOGRÁFICAS Y DOCUMENTALES}

Agencia Catalana del Agua - ACA (2008), Estudi de la Notorietat i Experiència d'ús de dispositius d'estalvi en aixetes. Informe Final, no publicado.

Aguilar, L.F. y Bustelo, M. (2010), “Gobernanza y evaluación: una relación potencialmente fructífera”, Gestión y Análisis de Políticas Públicas. Nueva Época, 4: 23-51.

Aguilar, L.F. (2007), "El aporte de la Política Pública y de la Nueva Gestión Pública a la gobernanza”, Revista Reforma y Democracia. Caracas: CLAD. En línea: <http:// www.clad.org/portal/publicaciones-del-clad/revista-clad-reforma-democracia/ articulos/039-octubre-2007/0057201> (consulta: 9 junio de 2011).

Alcantud, A. y Mazo, D. (2010), Impact Assessment Paper The "Install me!" Campaign. Communicative campaign within the Drought Context in Catalonia. Proyecto EUPOPP, no publicado.

Área Metropolitana de Barcelona - AMB (2011), "Dades Ambientals Metropolitanes 2010”. Dossier de Premsa: Barcelona: Entitat del Medi Ambient. En línea: <http:// www.amb.cat/c/document_library/get_file?p_I_id=6284\&folderld=236924\&na me=DLFE-8118.pdf $>$ (consulta: 9 junio 2011).

Área Metropolitana de Barcelona- AMB (2008), “Dades Ambientals Metropolitanes 2008”, Barcelona: Entitat del Medi Ambient. En línea: <http://www3.amb.cat/ ema/docum/dades_ambientals_08.pdf> (consulta: 8 marzo 2010).

INE, Instituto Nacional de Estadística (2009), Encuesta Hogares y Medio Ambiente 2008. Madrid: En línea: <http://www.ine.es/jaxi/menu.do?type=pcaxis\&path=\%2 Ft25/p500\&file=inebase\&L=0> (consulta: 17 enero 2011).

Mediterranean Water Scarcity and Drought Working Group - MED WS\&D WG (2007), 190 "Mediterranean water scarcity and drought report. Technical report on water 
scarcity and drought management in the Mediterranean and the Water Framework Directive", MED-EU Water Initiative / Water Framework Directive Joint Process. En línea: <http://www.emwis.net/topics/WaterScarcity/PDF/MedWSD_ FINAL_Edition> (consulta: 28 junio 2010).

\section{Textos legales}

Modificación del reglamento general del servicio metropolitano de suministro domiciliario de agua en el ámbito metropolitano, de 7 de febrero de 2008. Consejo Metropolitano de la EMSHTR (Boletín Oficial de la Provincia de Barcelona, 11 de febrero de 2008).

Decreto 93/2005, de 17 de mayo, de adopción de medidas excepcionales en relación al uso de los recursos hídricos (Diario Oficial de la Generalitat de Cataluña, 23 de mayo de 2005).

Decreto 84/2007, de 3 de abril, de adopción de medidas excepcionales y de emergencia en relación con el uso de los recursos hídricos (Diario Oficial de la Generalitat de Cataluña, 12 de abril de 2007).

Decreto 5/2009, de 13 de enero, por el cual se deroga el Decreto 84/2007, de 3 de abril, de adopción de medidas excepcionales y de emergencia en relación con el uso de recursos hídricos (Diario Oficial de la Generalitat de Cataluña, 16 de enero de 2009).

Moción Número 390-00021/08. Informe relativo al cumplimiento de la Moción 21/VII de la Sesión de Control del Parlamento de Cataluña (Boletín Oficial del Parlamento de Cataluña, 9 de junio de 2008: 65-68). 
